\title{
Effect of environmental tobacco smoke on cough in children with a history of tonsillectomy or adenoidectomy
}

\author{
Y. Chen*, D.C. Rennie+, L.A. Lockinger+, J.A. Dosman+
}

\begin{abstract}
Effect of environmental tobacco smoke on cough in children with a history of tonsillectomy or adenoidectomy. Y. Chen, D.C. Rennie, L.A. Lockinger, J.A. Dosman. OERS Journals Ltd 1998.
\end{abstract}

ABSTRACT: We examined the possible impact of tonsillectomy or adenoidectomy (T/ A) on the relationship between environmental tobacco smoke (ETS) exposure and respiratory outcomes.

This study was conducted in Humboldt, Saskatchewan, in 1993. The target population included all residents aged 6-17 yrs. Of the 1,019 eligible subjects, 892 participated $(88 \%)$. Estimates of ETS exposure were based on the reported smoking habits of the children's household members. We defined current cough as a positive response to the question: "Does this child usually have a cough?". Information also included morning cough, night cough and a history of T/A.

For children with no history of T/A, the prevalence of current cough was $8.9 \%$, $12.2 \%$ and $14.5 \%$ for those living in families with 0,1 , and $2+$ smokers respectively. The corresponding prevalence was $7.0 \%, 30.2 \%$ and $36.8 \%$ for children with history of T/A. Similar effects of ETS exposure were observed on morning cough and night cough. The results did not change significantly when we used various ETS measures and controlled for confounding factors. Compared to children living in nonsmoking families and without history of T/A, the adjusted odds ratio for children with a history of T/A was $7.19(\mathbf{p}<0.001)$ if they were living in families smoking $>20$ cigarettes $^{\cdot d a y} \cdot 1$ at home. The corresponding odds ratio was only $1.64(p=0.11)$ for children without a history of T/A.

We concluded that children living in smoking family were more likely to cough than those living in nonsmoking families and tonsillectomy or adenoidectomy increased the apparent influence of environmental tobacco exposure on cough. Eur Respir J 1998; 11: 1319-1323.
*Dept of Epidemiology and Community Medicine, Faculty of Medicine, University of Ottawa, Ottawa, Ontario, Canada. +Centre for Agricultural Medicine, Dept of Medicine, University of Saskatchewan, Saskatoon, Saskatchewan, Canada.

Correspondence: Y. Chen

Dept of Epidemiology and Community Medicine

Faculty of Medicine

University of Ottawa

451 Smyth Road

Ottawa

Ontario

Canada K1H 8M5

Fax: 16135625465

Keywords: Adenoidectomy, children, cough, passive smoking, tonsillectomy

Received: March 51997

Accepted after revision February 101998

Supported by a grant from the Saskatchewan Health Services Utilization and Research Commission, and by the National Health Research and Development Programme through a National Health Research Scholar award to Y. Chen.
The desirability of surgical removal of the tonsils and adenoids in children has been controversial among health professionals [1]. Although there has been a substantial decline in rates in recent years, tonsillectomy and adenoidectomy (T/A) are still commonly performed [1].

It has been demonstrated in previous studies that exposure to environmental tobacco smoke (ETS) increases the risk of a respiratory symptoms in children [2-5]. It is not known, however, whether children that have undergone removal of the tonsils or adenoids respond differently to ETS exposure as compared to the general population. The present report focuses on the difference in the influence of ETS exposure on cough in children with and without a history of T/A.

\section{Material and methods}

We conducted a study in the town of Humboldt, Saskatchewan, in 1993, which included cross-sectional, longitudinal and family components. The town of Humboldt has a stable population, a history of cooperation in previous health studies and a lack of industrial air pollution. The cross-sectional study of children and adolescents is a part of the study which has been described previously [6]. The target population included all town residents 6-17 yrs of age. All schools in the town (one high school and three elementary schools) provided lists of enrolled students aged 6-17 yrs. Subjects $<18$ yrs of age who were not attending school were identified by means of a total town canvass that was conducted for the adult portion of the study. Among the 1,019 eligible study subjects, 892 $(87.5 \%)$ participated in the study. Of the participants, $99.6 \%$ were of Caucasian background.

The questionnaires included information on: sociodemographic factors, alcohol consumption, exercise, history of allergy, individual and family history of pulmonary and cardiovascular diseases, and home environment, and were completed by the parents of children enrolled in the study. Subjects Š 12 yrs completed a second questionnaire by themselves on lifestyle topics including active smoking. Information about active smoking was not collected for children $<12$ yrs of age.

Current cough was defined as a positive response to the question: "Does this child usually have a cough?" Further, morning cough, day cough or night cough was positive if an affirmative response was given to the question: "Does your child usually cough at all on getting up, or first thing 
in the morning?" or "Does your child usually cough at all during the rest of the day or at night?" History of T/A was positive if there was an affirmative response to the question: "Did this child ever have an operation to remove the tonsils or adenoids?"

Estimates of household exposure to ETS were based on the reported smoking habits of the children's household members. We developed ETS measures as following: 1) maternal and paternal smoking status (nonsmoking, exsmoking and current smoking); 2) number of household smokers $(0,1$, and $2+) ; 3)$ current total daily cigarette consumption by household members; and 4) number of cigarettes smoked daily at home by household members. Males and females aged S12 yrs who reported smoking every day or almost every day, or had smoked at least 20 packs during their life time were defined as active smokers.

Primary analysis with simple frequency distributions and cross tabulations was conducted to examine the relationships between factors, including ETS exposure and prevalence of cough, and the Chi-square test was used to test significance of the relationships. The effect of household ETS exposure on cough was examined in children with and without history of T/A. A multiple logistic regression model was used to assess the interactive effects of ETS exposure and history of T/A on prevalence of cough before and after adjustment for covariates. Those unexposed to ETS and without history of T/A were defined as the reference category. Five indicator terms dealing with the joint effects of ETS exposure and history of T/A were built into the analysis. These indicated either the presence of each exposure and the absence of the other or the presence of joint exposure. The parameters of the models were estimated by the method of maximum likelihood.

\section{Results}

There were no significant differences between children from nonsmoking and smoking families in distributions of sex, age, number of bedrooms, heating fuel, home dampness, pet(s) and history of allergies. Compared to children from nonsmoking families, children from smoking families were more likely to live in older buildings and in homes other than single family dwellings, but in homes

Table 1. - Prevalence of cough according to various risk factors

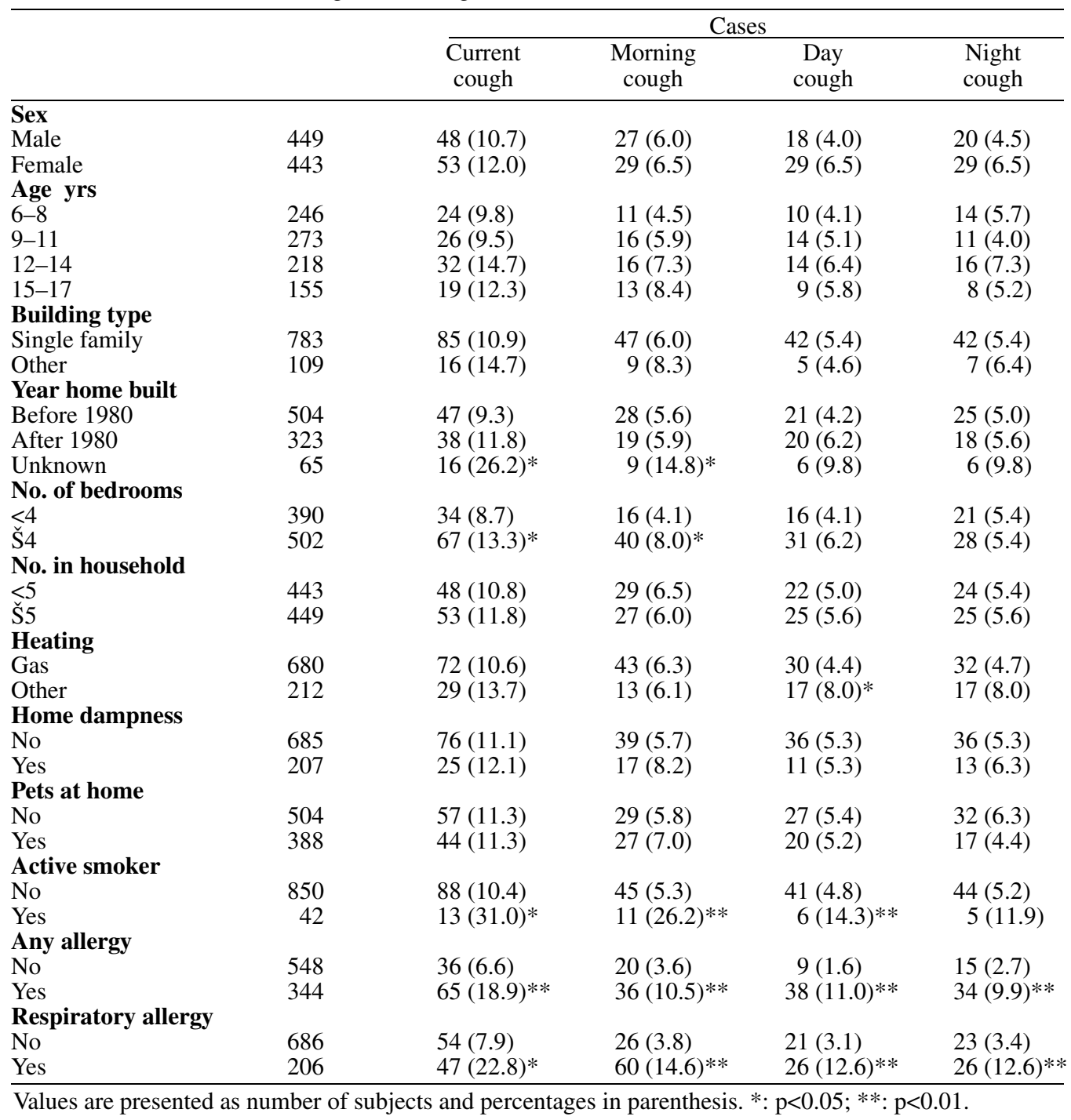


Table 2. - Prevalence of cough according to smoking variables

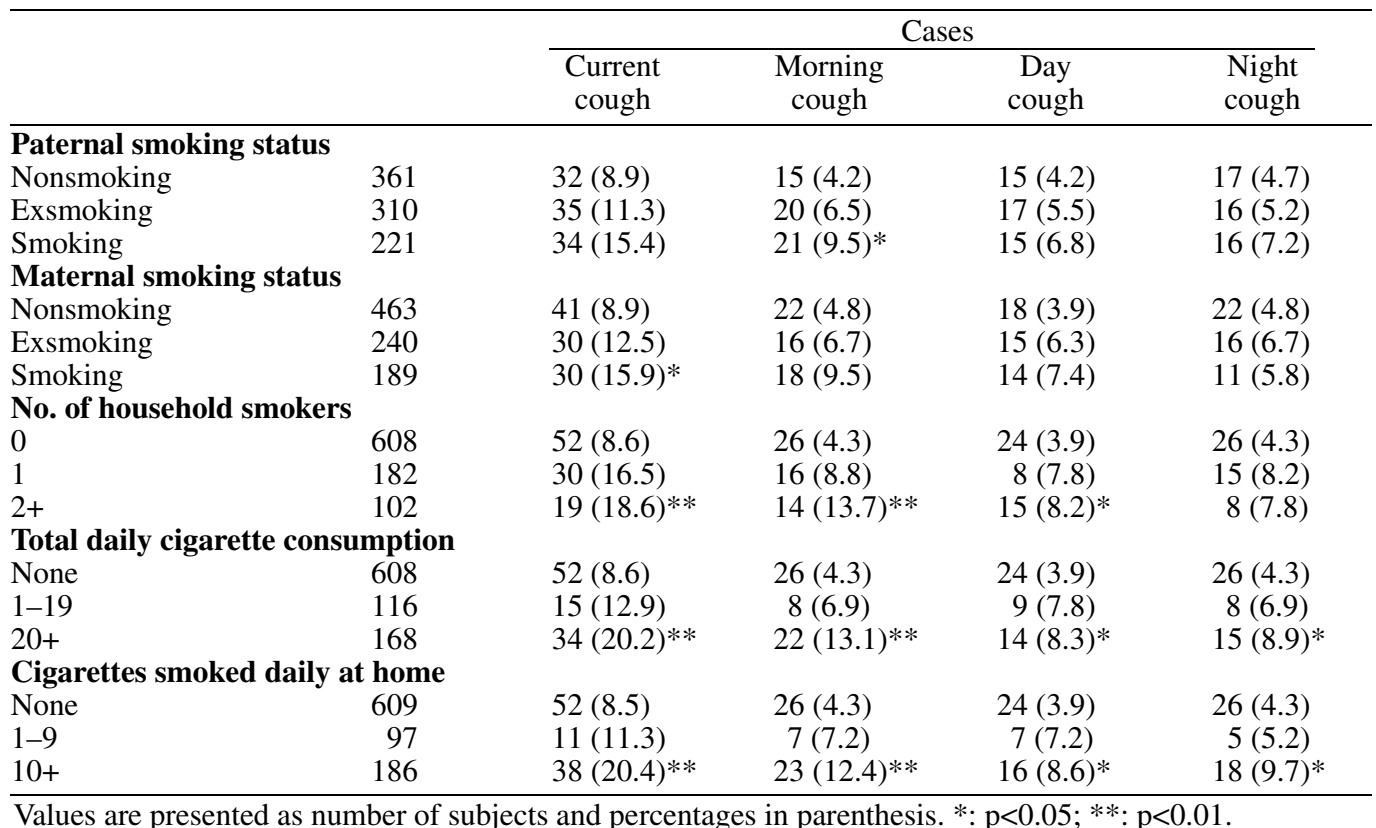

with fewer household members. Teenagers from families where there were smokers were more likely to be regular smokers (20.6\%), compared to those from nonsmoking families $(6.5 \%)$.

Current cough, morning cough, day cough and night cough occurred among $11.3,6.3,5.3$ and $5.5 \%$ of the study subjects respectively. Table 1 shows that current cough and morning cough were significantly related to the year that the home was built, number of bedrooms, active smoking and history of allergy. The prevalences of day cough and night cough were also higher in active smokers and in those with history of allergy. Sex, age, building type, number in household, home dampness and pets at home did not significantly predict the occurrence of cough.

Table 3. - Prevalence of current cough by cigarettes smoked daily at home by family members and history of tonsillectomy or adenoidectomy (T/A)

\begin{tabular}{lrrrrr}
\hline & \multicolumn{2}{c}{ Without T/A } & & \multicolumn{2}{c}{ With T/A } \\
\cline { 2 - 3 } \cline { 5 - 6 } $\begin{array}{l}\text { Cigarettes } \\
\text { per day }\end{array}$ & $\mathrm{n}$ & Cases & & $\mathrm{n}$ & Cases \\
None & 494 & $44(8.9)$ & & 115 & $8(7.0)$ \\
$1-9$ & 76 & $8(10.5)$ & & 21 & $3(14.3)$ \\
$10+$ & 146 & $21(14.4)$ & & 40 & $17(42.5)$ \\
$\chi^{2}$ & & 3.70 & & & 28.07 \\
p-value & & 0.157 & & & $<0.001$ \\
\hline
\end{tabular}

Cases are presented as number of subjects and percentages in parenthesis.
Table 2 shows that the prevalence of current cough increased with increasing number of household smokers and with daily cigarette consumption by household members other than the study subjects. The trend was similar for morning cough, day cough and night cough.

The children were classified into two groups based on history of T/A. Increasing numbers of household smokers, total daily cigarette consumption by household members and number of cigarettes smoked daily at home were significantly associated with increasing prevalence of current cough among children with a history of T/A. Paternal or maternal smoking also significantly predicted the occurrence of current cough. Among children without a history of T/A, the relationships between these smoking variables and current cough was weak and none of them reached statistical significance in this population. Table 3 presents only one smoking variable, namely the number of cigarettes smoked daily at home by household members.

A multiple logistic regression model was used to compare the joint effect of ETS exposure measured by cigarettes smoked daily at home by household members and history of T/A on current cough, before and after adjusting for the effects of other variables (e.g. year home was built, number in household, heating, active smoking and respiratory allergy). Table 4 shows the odds ratios for current cough in relation to each measure of ETS exposure and history of T/A. We defined the subjects living in

Table 4. - Unadjusted and adjusted* odds ratios (OR) and 95\% confidence intervals (CI) for current cough in relation to cigarettes smoked at home by household members and history of tonsillectomy or adenoi-dectomy (T/A)

\begin{tabular}{|c|c|c|c|c|}
\hline \multirow[b]{2}{*}{$\begin{array}{l}\text { Cigarettes } \\
\text { per day }\end{array}$} & \multicolumn{2}{|c|}{ Unadjusted OR $(95 \% \mathrm{CI})$} & \multicolumn{2}{|c|}{ Adjusted OR $(95 \% \mathrm{CI})$} \\
\hline & Without T/A & With T/A & Without T/A & With T/A \\
\hline None & 1.00 & $0.76(0.35-1.67)$ & 1.00 & $0.61(0.27-1.37)$ \\
\hline $1-9$ & $1.20(0.54-2.67)$ & $1.70(0.48-6.01)$ & $1.10(0.47-2.57)$ & $1.26(0.35-1.56)$ \\
\hline $10+$ & $1.72(0.99-3.00)$ & $7.56(3.76-15.21)$ & $1.64(0.90-3.00)$ & $7.19(3.19-16.23)$ \\
\hline
\end{tabular}

*: adjustment for: year that home was built (before 1980, after 1980, unknown); number of bedrooms ( $<4$, Š5); heating (gas, other); active smoking (no, yes); and respiratory allergy (no, yes). 
nonsmoking families and without history of T/A as the reference category. For children with a history of T/A, the adjusted odds ratio was $7.19(\mathrm{p}<0.001)$ if they were living in families smoking Š20 cigarettes day ${ }^{-1}$. The corresponding odds ratio was only $1.64(\mathrm{p}=0.11)$ among children without history of T/A.

The prevalences of morning cough, day cough and night cough were relatively low as compared to the prevalence of overall cough. The number of cases was too small, particularly in those with a history of T/A, to conduct further analysis.

\section{Discussion}

Previous epidemiological studies have demonstrated that household ETS exposure has an effect on frequency of cough in children [7-12]. It was estimated that the relative risk for cough related to household smoking ranged from 1.3-1.9, with an average of 1.4 [6]. Our estimation based on the data from all study subjects was similar to previous estimations and demonstrated an increased dose-effect relationship between ETS exposure (measured by number of household smokers and the amount they smoked) and current cough in children with a history of T/A, as compared to those without a history of T/A. Children who had tonsils and/or adenoids removed coughed more frequently in response to ETS exposure than did other children.

ETS exposure may increase the risk of T/A in children living in smoking families. SAID et al. [13] reported a relative risk of 1.6 based on the data from a cohort study. Case-control studies showed that children of smokers had a twofold risk of tonsillectomy for tonsillitis or adenoidectomy for recurrent otitis media $[14,15]$. ETS exposure is associated with respiratory infections and otitis media, which could result in the removal of tonsils or adenoids. The present study was not designed to examine the relationship between ETS exposure and the performance of T/ A. We collected the data of current ETS exposure and history of T/A and therefore the cause-effect of ETS exposure on T/A could not be evaluated in this study.

Tonsillectomy and adenoidectomy in children have generally been considered efficacious with regard to recurrent throat infections and to disease of the middle ear respectively, and are mainly used to reduce recurrent or chronic infection and obstruction of the upper respiratory tract [1]. Our data showed that the prevalence of current cough was not higher in children with a history of T/A than in those without a history of T/A who were living in nonsmoking families, which raised the possibility that T/A increases the susceptibility to cough in response to ETS exposure. However, in cross-sectional studies, it is difficult to establish a clear time sequence of events. There might be another explanation for the inter-relationship of T/A, ETS exposure and cough. T/A might have been performed because of increased cough. ETS exposure might have effects on both cough and T/A. However, it is hard to imagine, biologically, that the influence of ETS on T/A is modified by cough or the influence of cough on T/A is modified by ETS exposure.

The tonsils and adenoids are secondary lymphoid organs and part of the defence system of the human body [16]. The tonsils and adenoids are thought to play a role in the formation of immunoglobulins (IgA, G and M), inter- feron and antistreptolysin [17]. In a study of 46 children aged 3-10 yrs, CANTANI et al. [18] found that serum Ig and secretory $\operatorname{IgA}$ levels were significantly lower one month after T/A, which may predispose children to a constellation of disorders. KIRSTILA et al. [19] studied 25 young adults and found decreased levels of specific IgG antibodies and total IgM in saliva. Lower IgA levels were also found $6.6 \pm 2.1$ yrs after tonsillectomy in children aged 0.5-11 yrs [20]. However, other studies showed inconsistent results. LaL et al. [21] demonstrated that $\operatorname{IgG}, \operatorname{IgA}$ and $\operatorname{IgM}$ in patients with chronic tonsillitis before surgery were significantly higher than in controls, which might result from antigenic stimulation, and that only $\operatorname{IgG}$ showed a significant reduction after tonsillectomy. After studying 53 children 3-4 yrs after tonsillectomy, LENANDER-LUMIKARI $e t$ al. [22] suggested that the surgery did not weaken the humoral immune status of human saliva. Other researchers even suggested that tonsillectomy may improve the immune response [23]. Further studies are warranted to determine immunological mechanisms of predisposition to respiratory illness after T/A in response to ETS exposure.

Tonsillectomy or adenoidectomy may also physically alter the shape of the upper respiratory tract. Various agents may reach the airways more easily after removal of the tonsils or adenoids. Children with greater symptoms may be more prone to end up with surgery for tonsils and adenoids. However, these reasons do not explain the similar prevalence of cough in the children of nonsmokers with or without a history of T/A.

Allergic status could be an important confounding factor for the role of history of T/A on the relationship between ETS exposure and cough. Adjustment for history of allergy however, did not change the pattern of the relationship in children with and without a history of T/A substantially. Our data raises the possibility that surgical removal of the lymphoid tissue in oropharynx could have longterm implications for the health of children.

In conclusion, children living in smoking families were more likely to cough than those living in nonsmoking families and tonsillectomy or adenoidectomy increased the apparent influence of environmental tobacco smoke exposure on cough. The removal of tonsils and adenoids may have a negative impact on the respiratory system of children in response to environmental exposures.

\section{References}

1. Paradise JL. Tonsillectomy and adenoidectomy. In: Bluestone CD, Stool SE, Kenna MA, eds. Pediatric Otolaryngology (3rd edition). Philadelphia, W.B. Saunders Company, Vol. 2, 1996; pp. 1054-1065.

2. U.S. Department of Health and Human Services. The health consequences of involuntary smoking: a report of the Surgeon General. Washington, DC, U.S. Government Printing Office, 1986.

3. National Research Council. Environmental tobacco smoke: measuring exposures and assessing health effect. Washington, DC, National Academy Press, 1986.

4. National Cancer Institute. Respiratory health effect of passive smoking: lung cancer and other disorders. The report of the U.S. Environmental Protection Agency. NIH Publication No. 93-3605, 1993. 
5. DiFranza JR, Lew RA. Morbidity and mortality in children associated with the use of tobacco products by other people. Pediatrics 1996; 97: 560-568.

6. Chen Y, Rennie DC, Dosman JA. Influence of environmental tobacco smoke on asthma in nonallergic and allergic children. Epidemiology 1996; 7: 536-539.

7. Colley JRT. Respiratory symptoms in children and parental smoking and phlegm production. Br Med J 1974; 2: 201-204.

8. Lebowitz MD, Burrows B. Respiratory symptoms related to smoking habits of family adults. Chest 1976; 69: 4850 .

9. Bland M, Bewley BR, Pollard V, Banks MH. Effect of children's and parent's smoking on respiratory symptoms. Arch Dis Child 1978; 53: 100-105.

10. Dodge R. The effects of indoor pollution on Arizona children. Arch Environ Health 1982; 37: 151-155.

11. Ekwo EE, Weinberger MM, Lachenbruch PA, Huntley WH. Relationship of parental smoking and gas cooking to respiratory disease in children. Chest $1983 ; 84$ : 662668.

12. Charlton A. Children's coughs related to parental smoking. Br Med J 1984; 288: 1647-1649.

13. Said G, Zalokar J, Lellouch J, Patois E. Parental smoking related to adenoidectomy and tonsillectomy in children. $J$ Epidemiol Community Health 1978; 32: 97-101.

14. Hinton AE, Herdman RCD, Marin-Hirsch D, Saeed SR. Parental cigarette smoking and tonsillectomy in children. Clin Otolaryngol 1993; 18: 178-180.

15. Stahlberg MR, Ruuskanen O, Virolainen E. Risk factors for recurrent otitis media. Pediatr Infect Dis $J$ 1986; 5: $30-32$.

16. Wong HB. The problems of tonsils and adenoids. $J$ Singapore Paediatr Soc 1989; 31: 97-102.

17. Gray LP. Unilateral tonsillectomy - indications and results. J Laryngol Otol 1983; 97: 1111-1119.

18. Cantani A, Bellioni P, Salvinelli F, Businco L. Serum immunoglobulins and secretory $\operatorname{IgA}$ deficiency in tonsillectomized children. Ann Allergy 1986; 57: 413-416.

19. Kirstila V, Tenovuo J, Ruuskanen O, Suonpaa J, Meurman O, Vilja P. Longitudinal analysis of human salivary immunoglobulins, nonimmune antimicrobial agents, and microflora after tonsillectomy. Clin Immunol Immunopathol 1996; 80: 110-115.

20. Bock A, Popp W, Herkner KR. Tonsillectomy and the immune system: a long-term follow up comparison between tonsillectomized and non-tonsillectomized children. Eur Arch Otorhinolaryngol 1994; 251: 423-427.

21. Lal H, Sachdeva OP, Mehta HR. Serum immunoglobulins in patients with chronic tonsillitis. J Laryngol Otol 1984; 98: 1213-1216.

22. Lenander-Lumikari M, Tenovuo J, Puhakka HJ, et al. Salivary antimicrobial proteins and mutans streptococci in tonsillectomized children. Pediatr Dent 1992; 14: 8691.

23. Sainz M, Gutierrez F, Moreno PM, Munoz C, Ciges M. Changes in immunologic response in tonsillectomized children. I. Immunosuppression in recurrent tonsillitis. Clin Otolaryngol 1992; 17: 376-379. 